\title{
Mathematical Modelling of Human Amplifier Robot
}

\author{
Alper Kadir TANYILDIZI and Oğuz YAKUT
}

\begin{abstract}
Since the 1960s, several researches has been done on the conceptual design and active and passive control strategy of the lower extremity exoskeleton robot to achieve the most effective assistant exoskeleton robot in military and heavy industrial areas. In this study, a new active control strategy of two degrees of freedom exoskeleton robot was realized. Firstly, a double-pendulum (TDP) mechanism is used to demonstrate the human amplifier robot which follows and assist the healthy human low body movements. Then, mathematical model of this system is estimated. The numerical results are obtained and presented.
\end{abstract}

Keywords-Conceptual Design, Mathematical modeling of amplifier robot, Robotics.

\section{INTRODUCTION}

In recent years, wearable robots has been quite popular among research and projects in the field of robotics. Wearable robots are defined as robotic devices designed with joints and links to match the skeletal - muscle system and movement function of the human body when operator worn it $[1,2]$.

As a result of the transferring user's motivation desire to the robot via cognitive interaction network, the exoskeleton robot can support the movement activity of human and increase the physical strength (load carrying capacity, working time, etc.). Initially they were designed for military and rehabilitation applications [3], [4]. The term of exoskeleton robot is usually expressed as a mechanical device that increases the physical strength and power of a healthy human [3]. Accumulator design, controller strategy and performance evaluation criteria are three important steps to design an effective, portable, and safe exoskeleton robot [5]. The recognition of the human motion desire is very important for the efficient control of the exoskeleton robot used with the assistant of human motion. In recent years, many different exoskeleton robots have been designed by researchers for rehabilitation and military purposes [6], [7].

Comprehensive and large projects; besides the work of improving the mechanical design of the exoskeleton robot, the researchers emphasize the purpose and control of the robot in order to increase sensitivity, efficiency and comfort of the robot. The cognitive interaction between wearable robots and

Alper Kadir TANYILDIZI, PhD Student, Firat University, Mechatronics Engineering Department Turkey,

Assoc. Prof. Dr. Oğuz YAKUT is with Firat University, Mechatronics Engineering Department Turkey, humans and recognition of human desire are extremely important to effective and smooth control of exoskeleton. In addition, recent studies have shown that the correct recognition of the motion desire of the user makes the control of the exoskeleton easier and more successful in rehabilitation application. Hence, human-exoskeleton cognitive interaction and modeling is essential for all military, rehabilitation, assistant and augmentation applications. For this reason, there are three headings in the literature according to method of collecting data for human-robot interaction. These methods are

$\square$ Signals recorded from only human body

$\square$ Signals recorded only from the exoskeleton

Interaction force value measured

In the first method, it is the principle that the direct biological signals are recorded from the human directly and then human movement desire are determine via these signals. [8]. There is two types biological signal which is used human robot interaction studies. These are: the skin surface Electromyography (EMG) [9], [10]-[12] and the electroencephalogram (EEG) [13], [14]. Biomedical signal based interaction are used mostly rehabilitation exoskeleton in laboratory. Biomedical signal recorder devices are not useful for military exoskeleton robots. Because of biomedical sensors are effect environmental factors, vibration and impact are affected.

The second method is; is a model-based interaction strategy that not collects any biological signals from the human body and does not refer to the interaction forces [15]. In this method, motion analysis is based on the modeling of the human body and the exoskeleton robot. As it is known, this structure cannot be modeled without certain omissions. Such a model does not exactly reflect reality.

The third strategy used in this study is to design the humanrobot cognitive interaction via the interaction forces measured between the user and the exoskeleton. Some researchers measure this interaction force from the point of connection between the user and the robot, while others calculate the deformation rate of the elastic material placed in the robot link [16], [17]. A new distribute method are used in order to measure the pressure interaction between human and robot [18]. A healthy person worn a lower extremity exoskeleton robot named LOPES and the researchers tested their purposed method. The results showed that the interaction force between human and exoskeleton robot can be accurately calculated. Furthermore, this design is very comfort and safety for users 
and calculate interaction force in real time. The force based interaction method was used for HAL-5 [19]. Position of the center of gravity was calculated via floor reaction force (FRF) sensor to estimate the human- robot interaction. In [20], a force-sensing design was used to extract information about physical interaction.

It should also be noted that the accuracy of human robot interaction model and the comfortably are important factors when designing exoskeleton robots. The interaction strategy based on the principle of contact strength measurement is a common method for exoskeleton robots designed specifically for strengthening purposes [7, 21].

The rest of this paper is organized as follows. Section II presents conceptual design of our exoskeleton robot in detail. Section III presents mathematical modelling of double pendulum, which demonstrated interaction between human and exoskeleton leg. Section IV presents numerical results. Section V is conclusion.

\section{II.CONCEPTUAL DESIGN OF EXOSKELETON RoBOT}

In this study human-robot cognitive interaction is design via the interaction forces measured between the user and the exoskeleton. The force measuring sensors of the robot are positioned on the front and rear of the leg. Thus, when the leg starts to move, a reaction force will be generated in these sensors as the drivers of the robot will remain stationary. When attempting to move the human leg forward, the force sensors positioned on the front face will be activated. With a speed determined according to the magnitude of the measured reaction force, the driving units will try to move the robot limb forward. The drivers will continue to move the robot until the measured forces on the front face sensors reach zero. Likewise, if the force sensors on the rear side feel any pressure, the robot will move backwards. Thanks to this design, only one of the sensors (sensors on the front or rear face) among the sensors on the two sides of the robot can be activated at the same moment.

\section{Mathematical Model Of Human Leg And Human AMPLIFIER ROBOT}

There is a need for a mathematical model that reveals the interaction between human and robot. The kinematic diagram reflecting the physical model of human leg and exoskeleton is shown in Figure 1. The limb angles are denoted by $\theta \mathrm{i}$, the masses are by $\mathrm{mi}$, the lengths are by li, and centers of gravity are by Gi. The spring elements of k1 and k2 in the model represent force sensors that measure the reaction forces.

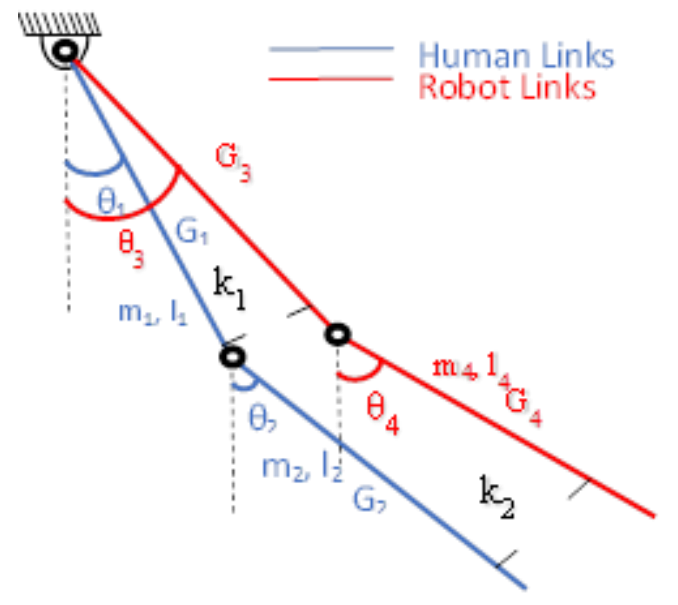

Fig. 1: Model of the robot and human limb

The Lagrange equations method is used when a mathematical model is constructed [22].

$$
\begin{aligned}
& L=T-V \\
& \frac{d}{d t}\left(\frac{\partial L}{\partial \dot{q}_{i}}\right)-\frac{\partial L}{\partial q_{i}}=\Im
\end{aligned}
$$

Where $\mathrm{T}$ denotes the kinetic and $\mathrm{V}$ the potential energy, and $\mathrm{cI}$ is the moment that must be applied to the joint.

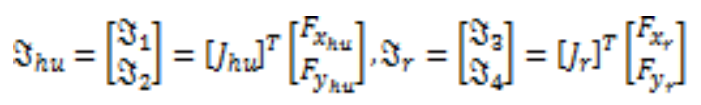

The Jhu, in the Equation 3, shows the Jacobian matrix of human leg, Fxhu is the force applied to the human heel in the $\mathrm{x}$ direction, and the Fyhu is the force applied to the human heel in the y direction. The Jacobean matrix of human leg and robot limbs are as follows:

$$
h_{h u}=\left[\begin{array}{cc}
l_{1} \cos \theta_{1} & l_{2} \cos \theta_{2} \\
-l_{1} \sin \theta_{1} & -l_{2} \sin \theta_{2}
\end{array}\right] \cdot J_{r}=\left[\begin{array}{cc}
l_{2} \cos \theta_{2} & l_{4} \cos \theta_{4} \\
-l_{2} \sin \theta_{a} & -l_{4} \sin \theta_{4}
\end{array}\right]_{(4)}
$$

The kinetic and potential energy expressions for human leg can be written as follows:

$$
\begin{gathered}
T_{h u}=\frac{1}{2} m_{1} V_{G_{1}}^{2}+\frac{1}{2} I_{1} \theta_{1}^{2}+\frac{1}{2} m_{2} V_{G_{2}}^{2}+\frac{1}{2} I_{2} \theta_{2}^{2} \\
V_{h u}=m_{2} g \frac{I_{2}}{2}\left(1-\cos \theta_{2}\right)+m_{2} g\left[\left(I_{1}+\frac{I_{2}}{2}\right)-\left(I_{1} \cos \theta_{2}+\frac{I_{2}}{2} \cos \theta_{2}\right)\right](6)
\end{gathered}
$$

Lagrange function can be obtained from here as follows:

$$
\begin{aligned}
& L_{h u}=\frac{1}{6} m_{1} l_{1} \theta_{1}^{2}+\frac{1}{2} m_{2} l_{1} \theta_{1}^{2}+\frac{1}{2} m_{2} l_{1} l_{2} \theta_{1} \theta_{2} \cos \left(\theta_{2}-\theta_{1}\right)+ \\
& \frac{1}{6} m_{2} l_{2} \theta_{2}^{2}-m_{1} g \frac{l_{1}}{2}\left(1-\cos \theta_{1}\right)-m_{2} g l_{2}\left(1-\cos \theta_{1}\right)- \\
& m_{2} g \frac{\mathbb{l}_{2}}{2}\left(1-\cos \theta_{2}\right)
\end{aligned}
$$

The obtained Lagrange function can be written in Equation 
(2) and written in the form of a matrix as follows after the necessary mathematical operations.

$$
\begin{aligned}
& {\left[\begin{array}{cc}
\frac{1}{a} m_{1} l_{1}^{2}+m_{2} l_{1}^{2} & \frac{1}{2} m_{2} l_{1} l_{2} \cos \left(\theta_{2}-\theta_{1}\right) \\
\frac{1}{2} m_{2} l_{1} l_{2} \cos \left(\theta_{2}-\theta_{1}\right) & \frac{1}{a} m_{2} l_{2}^{2}
\end{array}\right]\left[\begin{array}{l}
\theta_{1} \\
\theta_{2}
\end{array}\right]+} \\
& {\left[\begin{array}{cc}
-\frac{1}{2} m_{2} l_{1} l_{2} \sin \left(\theta_{2}-\theta_{1}\right) \theta_{2}^{2} \\
\frac{1}{2} m_{2} l_{1} l_{2} \sin \left(\theta_{2}-\theta_{1}\right) \theta_{1}^{2}
\end{array}\right]+\left[\begin{array}{c}
l_{1} g \sin \theta_{1}\left(\frac{m_{1}}{2}+m_{2}\right) \\
m_{2} g \frac{l_{2}}{2} \sin \theta_{2}
\end{array}\right]=} \\
& {\left[\begin{array}{ll}
l_{1} \cos \theta_{1} & -l_{1} \sin \theta_{1} \\
l_{2} \cos \theta_{2} & -l_{2} \sin \theta_{2}
\end{array}\right]\left[\begin{array}{l}
F_{x_{h u u}} \\
F_{y_{\mathrm{hu}}}
\end{array}\right]}
\end{aligned}
$$

Similarly, these operations can also be performed for the robot's limbs and the following expressions can be obtained.

$$
\begin{aligned}
& {\left[\begin{array}{cc}
\frac{1}{a} m_{a} l_{a}^{2}+m_{4} l_{a}^{2} & \frac{1}{2} m_{4} l_{a} l_{4} \cos \left(\theta_{4}-\theta_{a}\right) \\
\frac{1}{2} m_{4} l_{a} l_{4} \cos \left(\theta_{4}-\theta_{a}\right) & \frac{1}{a} m_{4} l_{4}^{2}
\end{array}\right]\left[\begin{array}{l}
\theta_{a} \\
\theta_{4}
\end{array}\right]+} \\
& {\left[\begin{array}{cc}
-\frac{1}{2} m_{4} l_{a} l_{4} \sin \left(\theta_{4}-\theta_{a}\right) \theta_{4}^{2} \\
\frac{1}{2} m_{4} l_{a} l_{4} \sin \left(\theta_{4}-\theta_{a}\right) \theta_{a}^{2}
\end{array}\right]+\left[\begin{array}{c}
l_{a} g \sin \theta_{a}\left(\frac{m_{3}}{2}+m_{4}\right) \\
m_{4} g \frac{l_{4}}{2} \sin \theta_{4}
\end{array}\right]=} \\
& {\left[\begin{array}{ll}
l_{a} \cos \theta_{a} & -l_{a} \sin \theta_{a} \\
l_{4} \cos \theta_{4} & -l_{4} \sin \theta_{4}
\end{array}\right]\left[\begin{array}{l}
F_{x_{r}} \\
F_{y_{r}}
\end{array}\right]}
\end{aligned}
$$

\section{NUMERICAL SIMULATIONS AND INTERPRETATIONS}

Numerical values of parameters used in simulation studies are given in Table 1-2. These parameters were chosen were chosen taking into account the anatomy of the body of an adult individual. The motion equations are solved using the Runge Kutta method from the fourth order with the codes prepared in the MATLAB package program.

TABLE I

KINEMATIC PARAMETERS FOR THE NUMERICAL SOLUTION

\begin{tabular}{l|l|c|l|l}
\hline & $\begin{array}{l}\text { Human } \\
\text { Femoral }\end{array}$ & $\begin{array}{l}\text { Human } \\
\text { Tibial }\end{array}$ & $\begin{array}{l}\text { Robot } \\
\text { Femoral }\end{array}$ & $\begin{array}{l}\text { Robot } \\
\text { Tibial }\end{array}$ \\
\hline Mass $(\mathrm{kg})$ & $8 \mathrm{~kg}$ & $4 \mathrm{~kg}$ & $1 \mathrm{~kg}$ & $1 \mathrm{~kg}$ \\
\hline Length $(\mathrm{m})$ & $0,45 \mathrm{~m}$ & $0,43 \mathrm{~m}$ & $0,45 \mathrm{~m}$ & $0,43 \mathrm{~m}$ \\
\hline
\end{tabular}

TABLE II

SPRING AND DAMPING PARAMETERS

\begin{tabular}{l|l}
\hline $\mathrm{k} 1$ (1. Spring coefficient) & $5 \times 10^{3} \mathrm{~N} / \mathrm{m}$ \\
\hline $\mathrm{k} 2$ (2. Spring coefficient) & $5 \times 10^{3} \mathrm{~N} / \mathrm{m}$ \\
\hline $\mathrm{c} 1$ (1. Damping coefficient) & $0,1 \mathrm{~N} . \mathrm{s} / \mathrm{m}$ \\
\hline $\mathrm{c} 2$ (2. Damping coefficient) & $0,1 \mathrm{~N} . \mathrm{s} / \mathrm{m}$ \\
\hline
\end{tabular}

The reason for choosing these initial starting conditions is to try to make the most difficult movement of the double pendulum and robot pair. The motion equation of the human leg is defined in the form of a matrix as follows:

$$
M \theta+V=\mathfrak{g}+Q
$$

where, $\mathrm{M}$ is the inertia matrix containing masses; $\mathrm{V}$ is matrix containing the Coriolis-centrifugal and gravitational forces, $\Im$ is the moment matrix to be applied to in joints, and
$\mathrm{Q}$ is the generalized vector of outer moments.

The generalized outer moments include moments arising due to damping $\left(\mathrm{T}_{\mathrm{c}}\right)$ and the spring $\left(\mathrm{T}_{\mathrm{k}}\right)$ which will affect both the leg and the robot. These moments can be calculated as follows:

$$
\begin{aligned}
T_{c} & =\left[\begin{array}{l}
l_{1} c_{1}\left(\theta_{1}-\theta_{a}\right) \\
l_{2} c_{2}\left(\theta_{2}-\theta_{4}\right)
\end{array}\right] \\
T_{k} & =\left[\begin{array}{l}
l_{1} k_{1} \sin \left(\theta_{1}-\theta_{2}\right) \\
l_{2} k_{2} \sin \left(\theta_{2}-\theta_{4}\right)
\end{array}\right]
\end{aligned}
$$

The coefficient matrices in motion equation for human leg can be clearly written as follows:

$$
\begin{gathered}
M_{1}=\left[\begin{array}{cc}
\frac{1}{a} m_{1} l_{1}^{2}+m_{2} l_{1}^{2} & \frac{1}{2} m_{2} l_{1} l_{2} \cos \left(\theta_{2}-\theta_{1}\right) \\
\frac{1}{2} m_{2} l_{1} l_{2} \cos \left(\theta_{2}-\theta_{1}\right) & \frac{1}{a} m_{2} l_{2}^{2}
\end{array}\right] \\
V_{1}=\left[\begin{array}{c}
-\frac{1}{2} m_{2} l_{1} l_{2} \sin \left(\theta_{2}-\theta_{1}\right) \dot{\theta}_{2}^{2} \\
\frac{1}{2} m_{2} l_{1} l_{2} \sin \left(\theta_{2}-\theta_{1}\right) \dot{\theta}_{1}^{2}
\end{array}\right]+\left[\begin{array}{c}
l_{1} g \sin \theta_{1}\left(\frac{m_{1}}{2}+m_{2}\right) \\
m_{2} g \frac{l_{2}}{2} \sin \theta_{2}
\end{array}\right] \\
Q_{1}=-T_{c}-T_{k} \\
\mathfrak{J}_{h u}=\left[\begin{array}{l}
\mathfrak{J}_{1} \\
\mathfrak{J}_{2}
\end{array}\right]=\left[l_{h u}\right]^{T}\left[\begin{array}{l}
F_{x_{h u}} \\
F_{y_{h u}}
\end{array}\right]
\end{gathered}
$$

Although $\widetilde{\Im}_{\mathrm{i}}$ is defined, this matrix will be equal to zero as no load will be applied to the human leg. Similarly, these matrices for the robot limb can be written as follows:

$$
\begin{aligned}
& M_{2}=\left[\begin{array}{cc}
\frac{1}{a} m_{a} l_{a}^{2}+m_{4} l_{a}^{2} & \frac{1}{2} m_{4} l_{a} l_{4} \cos \left(\theta_{4}-\theta_{a}\right) \\
\frac{1}{2} m_{4} l_{a} l_{4} \cos \left(\theta_{4}-\theta_{a}\right) & \frac{1}{a} m_{4} l_{4}^{2}
\end{array}\right] \\
& V_{2}=\left[\begin{array}{c}
-\frac{1}{2} m_{4} l_{a} l_{4} \sin \left(\theta_{4}-\theta_{a}\right) \dot{\theta}_{4}^{2} \\
\frac{1}{2} m_{4} l_{a} l_{4} \sin \left(\theta_{4}-\theta_{a}\right) \dot{\theta}_{a}^{2}
\end{array}\right]+\left[\begin{array}{c}
l_{3} g \sin \theta_{a}\left(\frac{m_{3}}{2}+m_{4}\right) \\
m_{4} g \frac{l_{4}}{2} \sin \theta_{4}
\end{array}\right] \\
& Q_{2}=T_{c}+T_{k}+v
\end{aligned}
$$

The $\mathrm{v}$ vector which is within the matrix of generalized outer moment shows the control signals to be applied to the actuators in the hip and knee joints.

$$
\mathfrak{J}_{y}=\left[\begin{array}{l}
\mathfrak{J}_{a} \\
\mathfrak{J}_{4}
\end{array}\right]=\left[J_{\gamma}\right]^{T}\left[\begin{array}{l}
F_{x_{r}} \\
F_{y_{r}}
\end{array}\right]
$$

Thus, the acceleration equations can be written as follows:

$$
\begin{aligned}
& {\left[\begin{array}{l}
\theta_{1} \\
\theta_{2}
\end{array}\right]=M_{1}^{-1}\left(-V_{1}+Q_{1}\right)} \\
& {\left[\begin{array}{l}
\theta_{a} \\
\theta_{4}
\end{array}\right]=M_{2}^{-1}\left(-V_{2}+Q_{2}+\mathfrak{J}_{Y}\right)}
\end{aligned}
$$

In the numerical solutions, the initial conditions given in Table 4 are used. Spring constant is chosen as $5 \times 10^{3} \mathrm{~N} / \mathrm{m}$ for $\mathrm{k}_{1}$ and $\mathrm{k}_{2}$ springs. 
TABLE III

INITIAL CONDITIONS OF JOINTS

\begin{tabular}{l|c|c|c|c}
\hline & \multicolumn{2}{|c|}{ Human Link } & \multicolumn{2}{c}{ Robot Link } \\
\hline Position $\left(\boldsymbol{\theta}_{i 0}\right)($ degree $)$ & 45 & -45 & 45 & -45 \\
\hline Angular Velocity $\left(\boldsymbol{\theta}_{i 0}\right)(\mathrm{m} / \mathrm{s})$ & 0 & 0 & 0 & 0 \\
\hline
\end{tabular}

In the simulations, it is aimed to make the exoskeleton robot go to the joint angle to zero from the starting initial position with gravity effect. Change of Joint angles of human leg and exoskeleton robot, error of the joints and spring force against time are presented Fig.1,2,3 respectively.
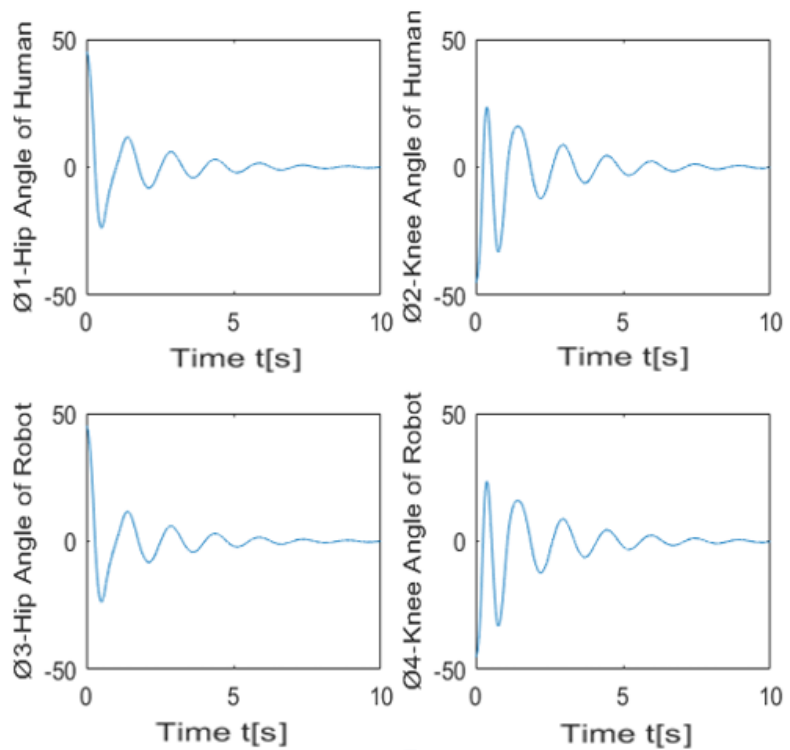

Fig.2 Hip and Knee Angles of Human and Robot
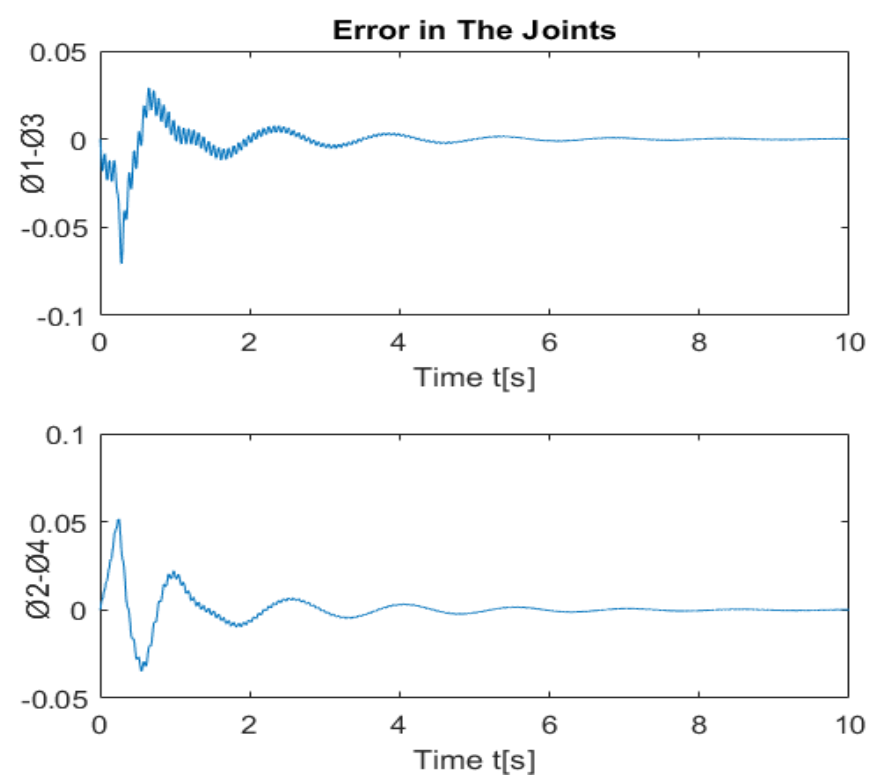

Fig. 3 Error in the joints angle
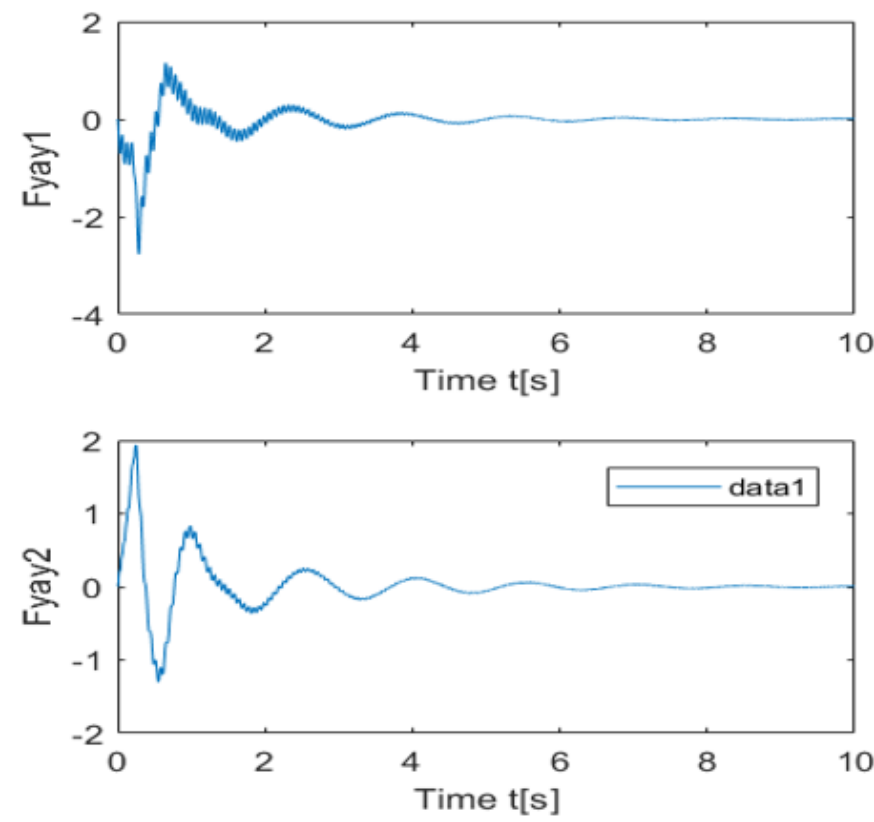

Fig. 4 Spring forces

\section{V.CONCLUSIONS}

This paper presents the conceptual design and mathematical modelling of a wearable lower limb exoskeleton developed to augment healthy individuals such as soldiers. Humanexoskeleton robot interaction was supported via force sensors. Force sensor was placed between the leg and the robot limb to provide the interaction between the human and exoskeleton robot. This pressured interaction between the robot limb and the human knee was modeled as a spring element and mathematical motion equations were obtained. Numerical results are obtained for uncontrolled release motion are simulated. According to the obtained results, it is obvious that the estimated mathematical model is useful to analyze motion of exoskeleton robot. Our aim to establish a real prototype of this system and controller design for system in the future studies and verify these results with real-time control.

\section{ACKNOWLEDGMENT}

The subject of this article, which is Alper TANYILDIZI's doctoral thesis, was supported by Firat University Scientific Research Projects Management Unit within the scope of $\mathrm{PhD}$ Thesis Project number MF-17.12

\section{REFERENCES}

[1] W., Huo, S., Mohammed, J. C., Moreno, and Y., Amirat, Lower Limb Wearable Robots for Assistance and Rehabilitation: A State of the Art, IEEE Systems Journal, Vol. 10, No. 3, September 2016

[2] J. L. Pons, Wearable Robots: Biomechatronic Exoskeletons. New York, NY, USA: Wiley, 2008 https://doi.org/10.1002/9780470987667

[3] A. M. Dollar and H. Herr, "Lower extremity exoskeletons and active orthoses: Challenges and state of the art," IEEE Trans. Robot., vol. 24, no. 1 , pp. $144-158$, Feb. 2008 https://doi.org/10.1109/TRO.2008.915453.

[4] M. Vukobratovic, "Humanoid robotics-Past, present state, future," in Proc. 4th Serbian-Hungarian Joint Symp. Intell. Syst., 2006, pp. 13-31. 
[5] R. E. Cowanet al., "Recent trends in assistive technology for mobility," J. NeuroEng. Rehabil. , vol. 9, no. 20, pp. 1-8, Apr. 2012.

[6] S. Mohammed, Y. Amirat, and H. Rifai, "Lower-limb movement assistance through wearable robots: State of the art and challenges," Adv. Robot., vol. 26, no. 1/2, pp. 1-22, 2012. https://doi.org/10.1163/016918611X607356

[7] L. Marchal-Crespo and D. J. Reinkensmeyer, "Review of control strategies for robotic movement training after neurologic injury," J. NeuroEng. Rehabil., vol. 6, no. 20, pp. 1-15, 2009 https://doi.org/10.1186/1743-0003-6-20.

[8] J. L Pons, "Rehabilitation exoskeletal robotics," IEEE Eng. Med. Biol. Mag., vol. 29, no. 3, pp. 57-63, May/Jun. 2010. https://doi.org/10.1109/MEMB.2010.936548

[9] H. Kawamoto, S. Lee, S. Kanbe, and Y. Sankai, "Power assist method for HAL-3 using EMG-based feedback controller," in Proc. IEEE Int. Conf. Syst., 2003, pp. 1648-1653. https://doi.org/10.1109/ICSMC.2003.1244649

[10] C.Fleischer, A.Wege, K.Kondak, and G.Hommel, "ApplicationofEMG signals for controlling exoskeleton robots," Biomed. Tech. Biomed. Eng., vol. 51, no. 5/6, pp. 314-319, Dec. 2006. https://doi.org/10.1515/BMT.2006.063

[11] C. Fleischer, C. Reinicke, and G. Hummel, "Predicting the intended motion with EMG signals for an exoskeleton orthosis controller," in Proc. IEEE Int. Conf. Robot. Auton. Syst., 2005, pp. 2029-2034. https://doi.org/10.1109/IROS.2005.1545504

[12] C. Fleischer and G. Hummel, "Embedded control system for a powered leg exoskeleton," in Embedded Systems-Modeling, Technology, and Applications. New York, NY, USA: Springer-Verlag, 2006, pp. 177185. https://doi.org/10.1007/1-4020-4933-1_19

[13] Y. Wang and S. Makeig, "Predicting intended movement direction using EEG from human posterior parietal cortex," in Proc. 5th Int. Conf. Found. Augmented Cognition. Neuroergonomics Oper. Neurosci., vol. 5638, Lecture Notes in Computer Science, 2009, pp. 437-446. https://doi.org/10.1007/978-3-642-02812-0_52

[14] E. Lew, R .Chavarriaga, S. Silvoni, and J.D.R.Millán, "Detectionofselfpaced reaching movement intention from EEG signals," Front Neuroeng., vol. 5, no. 13, pp. 1-17, Jul. 2012.

[15] H. Kazerooni and R. Steger, "The Berkeley lower extremity exoskeletons," Trans. ASME J. Dynam. Syst. Meas. Control, vol. 128, pp. 14-25, Mar. 2006 https://doi.org/10.1115/1.2168164

[16] A. Valiente, "Design of a Quasi-Passive Parallel Leg Exoskeleton to Augment Load Carrying for Walking," M.S. thesis, Dept. Mech. Eng., Massachusetts Inst. Technol., Cambridge, U.K., Aug. 2005.

[17] H. Kawamoto and Y. Sankai, "Power assist method based on phase sequence and muscle force condition for HAL," Adv. Robot., vol. 19, no. 7, pp. 717-734, 2005. https://doi.org/10.1163/1568553054455103

[18] M. De Rossi et al., "Sensing pressure distribution on a lower-limb exoskeleton physical human-machine interface," Sensors, vol. 11, no. 1, pp. 207-227, Jan. 2011. https://doi.org/10.3390/s110100207

[19] K. Suzuki, G. Mito, H. Kawamoto, Y. Hasegawa, and Y. Sankai, "Intention-based walking support for paraplegia patients with robot suit HAL," Adv. Robot., vol. 21, no. 12, pp. 1441-1469, 2007.

[20] A.J.del-Ama,J.C.Moreno,A.Gil-Agudo,A.de-los-Reyes,andJ.L.Pons, "Online assessment of human-robot interaction for hybrid control of walking," Sensors, vol. 12, no. 1, pp. 215-225, Jan. 2012 https://doi.org/10.3390/s120100215.

[21] S. K. Banala, S. K. Agrawal, S. H. Kim, and J. O. Scholz, "Novel gait adaptation and neuromotor training results using an active leg exoskeleton,'IEEE/ASMETrans.Mechanicals.Mechatronics,vol.15,no.2, pp. 216-225, Apr. 2010. https://doi.org/10.1109/TMECH.2010.2041245

[22] Mukul K. Gupta, Kamal Bansal, Arun K. Singh, Mass and Length Dependent Chaotic Behavior of a Double Pendulum, Third International Conference on Advances in Control and Optimization of Dynamical Systems March 13-15, 2014. Kanpur, India. 